\title{
The Lymphocyte Transformation Test for Borrelia Detects Active Lyme Borreliosis and Verifies Effective Antibiotic Treatment
}

\author{
Volker von Baehr ${ }^{1}$, Cornelia Doebis ${ }^{1}$, Hans-Dieter Volk ${ }^{2}$, Rüdiger von Baehr ${ }^{1, *}$ \\ ${ }^{1}$ Institute for Medical Diagnostics, Immunology Department, Nicolaistrasse 22, 12247 Berlin \\ ${ }^{2}$ Institute for Medical Immunology, Charité University Medicine Berlin, Campus Mitte, Charitéplatz 1, 10117 Berlin
}

\begin{abstract}
Borrelia-specific antibodies are not detectable until several weeks after infection and even if they are present, they are no proof of an active infection. Since the sensitivity of culture and PCR for the diagnosis or exclusion of borreliosis is too low, a method is required that detects an active Borrelia infection as early as possible. For this purpose, a lymphocyte transformation test (LTT) using lysate antigens of Borrelia burgdorferi sensu stricto, Borrelia afzelii and Borrelia garinii and recombinant $\mathrm{OspC}$ was developed and validated through investigations of seronegative and seropositive healthy individuals as well as of seropositive patients with clinically manifested borreliosis. The sensitivity of the LTT in clinical borreliosis before antibiotic treatment was determined as $89,4 \%$ while the specificity was $98,7 \%$. In 1480 patients with clinically suspected borreliosis, results from serology and LTT were comparable in $79.8 \%$ of cases. $18 \%$ were serologically positive and LTT-negative. These were mainly patients with borreliosis after antibiotic therapy. $2.2 \%$ showed a negative serology and a positive LTT result. Half of them had an early erythema migrans. Following antibiotic treatment, the LTT became negative or borderline in patients with early manifestations of borreliosis, whereas in patients with late symptoms, it showed a regression while still remaining positive. Therefore, we propose the follow-up monitoring of disseminated Borrelia infections as the main indication for the Borrelia-LTT.
\end{abstract}

Keywords: Borrelia serology, borreliosis, diagnostics, immune response, lymphocyte transformation test, $\mathrm{T}$ cells.

\section{INTRODUCTION}

Lyme borreliosis is the most common disease transmitted by tick bite. Lyme borreliosis first manifests locally on the skin at the site of the tick bite and then systemically, possibly affecting one or more organs such as the skin, joints, muscles, sense organs, nervous system and heart. In the latter case, early (stage I and II) and late (stage III) manifestations can be distinguished (1). Lyme borreliosis should be diagnosed by history and clinical symptoms. If the clinical symptoms are clear, laboratory diagnostics are of secondary importance only. The difficulty is, however, that the tick bite often goes unnoticed and the erythema migrans does not necessarily occur or is not noticed. In these cases, the requirement for early antibiotic treatment of borreliosis to prevent the complications of systemic dissemination of the pathogen, particularly of late borreliosis, cannot be met.

The symptoms associated with the systemic phase of Lyme borreliosis can be highly varied and ambiguous. In these cases, the detection of Borrelia-specific antibodies (serological laboratory diagnosis) becomes important for the diagnosis and treatment decision. The necessarily high quality demands cannot yet be completely fulfilled by Borrelia serology due to the following reasons: 1) Borrelia-specific

\footnotetext{
*Address correspondence to this author at the Institut für Medizinische Diagnostik Berlin, Nicolaistraße 22, 12247 Berlin, Germany;

Tel: +49-30-77001220; Fax: +49-30-77001236;

E-mail: v.baehr@imd-berlin.de
}

IgM antibodies, and IgG antibodies in particular, cannot be detected until several weeks after infection [1,2].Seronegative cases with late stage Lyme borreliosis have also been recently described [3]. But these are becoming more rare with the increasing quality of the assays following the introduction of recombinant Borrelia antigens. 2) The heterogeneity of Borrelia species and strains within a species requires a polymorphism of the Borrelia-specific protein antigens $[4$, 5]. This is a difficult problem for the sensitivity of Borrelia serology. 3) IgM antibodies against Borrelia OspC may be of the nonspecific type $[4,5]$. 4) A positive serological finding alone is not proof of a current active Borrelia infection [1, 4, 5]. 5) Borrelia serology is not suitable for the monitoring of therapy and evaluation of progress as $\operatorname{IgG}$ and $\operatorname{IgM}$ antibodies may persist for years after borreliosis has been cured [6].

The direct detection of Borrelia by culture or PCR has a high diagnostic value in the case of a positive result, but a negative result does not rule out Lyme borreliosis $[4,5]$.

There is currently no method available which, in addition to the serology, answers the question as to whether a specific case is a status post Borrelia infection or active borreliosis.

Each humoral immune response to an infection requires a specific cellular immune response with clonal proliferation of various antigen-specific lymphocyte subpopulations. Of central importance here are antigen-specific $\mathrm{T}$ helper lymphocytes $\left(\mathrm{CD} 4^{+} \mathrm{T}_{\mathrm{H}}\right.$ cells). In addition to effector $\mathrm{T}$ cells, long-lived $\mathrm{T}$ and $\mathrm{B}$ memory lymphocytes are formed. In the presence of antigen-presenting cells and protein antigens, 
specific $\mathrm{CD}^{+} \mathrm{T}$ memory cells also proliferate in vitro. The lymphocyte transformation test (LTT), also known as the lymphocyte proliferation or lymphocyte activation test, is based on this principle [7]. Shortly after the discovery of B. burgdorferi, it was demonstrated that blood lymphocytes from Lyme borreliosis patients proliferate in the presence of Borrelia lysates $[8,9]$. Published data on the diagnostic value of the Borrelia-LTT, especially in seronegative patients, can be found beginning in 1988 [10-17]. However, false positive LTT reactions have also been described [18-20]. Important for the motivation of our investigations presented here were observations that positive LTT reactions of blood lymphocytes to Borrelia antigens declined significantly or were negative after antibiotic treatment of Lyme borreliosis $[12,14$, $16,21]$. This leads to the hypothesis that Borrelia-specific $T$ helper cells circulate in the blood in detectable numbers only during an active immune response against Borrelia and persist in a non-florid infection in lymphoid organs.

Using improved cell culture and measurement techniques as well as our own experience in the development of antigenspecific LTT applications, we will seek to answer using reevaluation of patient data the following questions:

1. Is there a correlation between the results of Borrelia serology and Borrelia-LTT?

2. What are the Borrelia-LTT results in clinically healthy seropositive subjects?

3. Is it possible to obtain an indication of the respective species involved from the LTT reactions to antigens of the three Borrelia species?

4. Are Borrelia-LTT results influenced by antibiotic treatment?

5. How high are the sensitivity and specificity of the Borrelia-LTT?

\section{MATERIALS AND METHODS}

\section{Subjects and Patients}

All laboratory tests were performed in the course of routine diagnostic procedures that were applied to prove or to exclude borreliosis, respectively.

The statistical re-evaluation was performed retroactive considering the laboratory data and the documented clinical data by the doctors.

The seronegative control group $(\mathrm{n}=160)$ included 120 clinically healthy subjects (blood donors) and 40 patients with autoimmune diseases (rheumatoid arthritis, lupus erythematosus viszeralis, Hashimoto's thyroiditis, diabetes mellitus type 1) (86 men, 74 women, aged 18 to 72 years).

The seropositive group included patients with clinical borreliosis $(\mathrm{n}=94)$; erythema migrans $(\mathrm{n}=28)$, acute monoarthritis $(\mathrm{n}=14)$, Bannwarth's syndrome $(\mathrm{n}=6)$, migrating arthromyalgias $(\mathrm{n}=34)$, facial palsy $(\mathrm{n}=5)$, acute neuroborreliosis $(\mathrm{n}=7)$ as well as clinically healthy subjects $(\mathrm{n}=$ 48) including forest workers and hunters, persons working full time in agriculture and horticulture and hobby gardeners (34 men, 14 women; aged 24-72 years).
Borrelia serology and Borrelia-LTT were investigated in parallel in patients with unknown serology but clinical diagnosis of suspected Lyme borreliosis $(\mathrm{n}=1480)$.

Patients with clinical borreliosis with positive or unknown serology were used for LTT progression studies.

The blood of 20 healthy persons was used for the preliminary finding of appropriate antigen concentrations for the LTT.

\section{Borrelia Serology}

For antibody determination, recombinant Borrelia $\operatorname{IgG}$ and IgM ELISA (Mikrogen, Munich, Germany) and the IgG and IgM immunoblot / recombinant immunoblot (Mikrogen) were used. The immunoblot contains the following specific recombinant Borrelia antigens: p100, p41 int (B. afzelii., B. garinii.), p41 (B. sensu stricto), p39, p31, p25, p18, VslE.

The tests and evaluation of the results were performed according to the working instructions of the manufacturer. The results for the ELISA test are given as "negative" or "positive". Only the results of the Borrelia immunoblot were evaluated for the classification of the subjects / patients as "seronegative", "borderline" or "seropositive".

\section{Borrelia Antigens}

Purified lyophilized lysate antigens generated from day 6 cultures of Borrelia sensu stricto (strain LW2 isolated from skin biopsy), Borrelia afzelii (strain Pko, DSM No. 16073) and Borrelia garinii (strain PBi isolated from CSF, Pettenkofer Institute, Munich) were kindly provided by Seramun, Heidesee, Germany. Seramun determined the protein content in purified lysate using the BCA Protein Assay Kit (Pierce, Prod. No: 23225). Each lysate contains, among others, the following antigens: p83/100; p58; $443 ; \mathrm{p} 41 ; \mathrm{p} 39$; p30; p22-25; p21; p19; p17. Antigens were tested by using IgG immunoblot (Fig. 1, supplementary data). All lysate antigens used in the LTT test are tested by Seramun with 8 positive and negative control sera in the solid phase IgG and IgM ELISA. Only those lysates were used for the LTT test that reacted with positive control sera only, and not with negative control sera.

Recombinant OspC of B. afzelii (host cell E. coli TG) was provided by Micromun, Greifswald, Germany. The protein is purified through Ni Sepharose (Amersham Bioscience) and purity testing was performed by Microimun using polyacrylamide gel electrophoresis.

\section{Lymphocyte Transformation Test (Syn. Lymphocyte Proliferation Test)}

$10 \mathrm{ml}$ of heparinized venous blood were obtained under sterile conditions and processed by density gradient centrifugation to obtain peripheral blood mononuclear cells $(85-95 \%$ lymphocytes and $5-15 \%$ monocytes) as described elsewhere [21]. After washing the cells with PBS (PAA Laboratories, Linz, Austria), the cell pellet was resuspended to obtain a cell count of $1 \times 10^{6} / \mathrm{ml}$ in cell culture medium (RPMI 1640; PAA) supplemented with $2 \mathrm{mM}$ L-glutamine (PAA), 100 $\mu \mathrm{g} / \mathrm{ml}$ gentamicin (PAA) and $5 \%$ autologous serum. To improve antigen presentation and suppression of nonspecific 
cell activation, recombinant interferon- $\alpha$ (rIFN $\alpha 2 b$, Schering-Plough, Kenilworth, USA) was added to all assays at a concentration of $125 \mathrm{U} / \mathrm{ml}$ (23). To inhibit nonspecific activating effects caused by the lipoids and lipoproteins contained in the Borrelia lysate antigen preparations, polymyxin $\mathrm{B}$ (PAA) is used in a final concentration of $5 \mu \mathrm{g} / \mathrm{ml}$. All assays were performed in triplicates. Cell stimulation was performed by using $2 \times 10^{5}$ cells/well in a 96-well microplate (Nunclon, Wiesbaden, Germany) for 6 days at $37^{\circ} \mathrm{C}$ and $5 \%$ $\mathrm{CO}_{2}$ atmosphere. Cells were labeled with ${ }^{3} \mathrm{H}$-thymidine (1 $\mu \mathrm{Ci} / \mathrm{ml}$, Hartmann Analytics, Braunschweig, Germany) 12 hours prior to cell harvest. Cell harvest was performed on glass fiber filters (Wallac, Lund, Sweden) using a cell harvester (Wallac). The incorporated ${ }^{3} \mathrm{H}$-thymidine activity was measured as "counts per minute" (cpm) with a solid phase beta counter (Wallac). Mean value of the triplicates are used for analysis. The results for each antigen are given as a stimulation index (SI; ratio of cpm of the culture with and w/o test antigen).

To demonstrate the functional integrity of the immune cells, positive controls were performed in parallel. As an antigen-presenting cell-dependent positive control a recall antigen mixture of tetatoxoid (GlaxoSmithKline, Munich, Germany), influenza split antigen (GlaxoSmithKline) and cytomegalovirus antigen (Abbott, Wiesbaden, Germany) is used. As mitogen control the nonspecific cell stimulator pokeweed mitogen (PWM, PAA) is added in a concentration of $1.5 \mu \mathrm{g} / \mathrm{ml}$. The test evaluation is performed only if antigen control reaches SI $>5$ and mitogen control reaches $\mathrm{SI}>20$.

For the assessment of the Borrelia-LTT we used the following cut-offs:

$\mathrm{SI}<3=$ negative test result,

$\mathrm{SI}>5=$ positive test result,

SI 3 to $5=$ weak positive test result.

For the validation of the Borrelia-LTT a test result is considered positive if at least two of the four Borrelia antigens show SI values of 3 and higher.

\section{Statistics}

The sensitivity of the LTT was evaluated by taking the percentual number of LTT positive patients in relation to all seropositive patients with clinical borreliosis. The specificity was calculated by taking the relative number of LTT negative results in a group of seronegative clinical healthy subjects.

Statistical analysis for the LTT validation results was performed by using the Chi-square test (Prism 5, GraphPad software, La Jolla, USA).

\section{RESULTS}

\section{Determining the Appropriate Test Dose of Antigen for the Borrelia-LTT}

To validate the antigen specificity of the Borrelia-LTT, blood samples were used from 20 clinically healthy Borreliaseronegative volunteers. Isolated PBMC were tested in a dilution series $(12,8,4,2,1,0.5 \mu \mathrm{g} / \mathrm{ml})$ of the 4 Borrelia test antigens in the LTT assay. Using this setting the highest antigen concentration for the Borrelia-LTT which did not give any positive (SI from 3 ) or borderline $(\mathrm{SI}>2<3$ ) LTT result was determined. The working concentrations per assay calculated in this manner contained $1 \mu \mathrm{g}$ of protein for the lysate antigen of B. sensu stricto, $2 \mu \mathrm{g}$ each of the lysate antigens of B. afzelii and B. garinii, and $0.5 \mu \mathrm{g}$ for rOspC. All further tests were performed with the given antigen concentrations.

\section{Sensitivity and Specificity of the Borrelia-LTT}

The results of the validation studies for the Borrelia-LTT are summarized in Table $\mathbf{1}$.

In 160 seronegative subjects/patients (120 healthy seronegative and 40 patients with autoimmune diseases), a specificity of $98,7 \%$ was found if the borderline results of SI $>2<$ 3 were evaluated as negative. Weak false positive reactions $(\mathrm{SI}>3<5)$ were detected in only 2 cases. No differences were found between clinically healthy subjects and patients with autoimmune diseases.

In 94 seropositive patients with clinical borreliosis before antibiotic treatment, the sensitivity of the Borrelia-LTT (SI $>3$ to 28 ) was $89.4 \%$. Ten proved to be negative $(10,6 \%)$. From the 13 patients with a typical clinical picture of neuroborreliosis, 10 showed positive results in the LTT. Two out of 5 patients with facial palsy and 1 out of 7 patients with

Table 1. Results of Validation Studies of the Borrelia-LTT

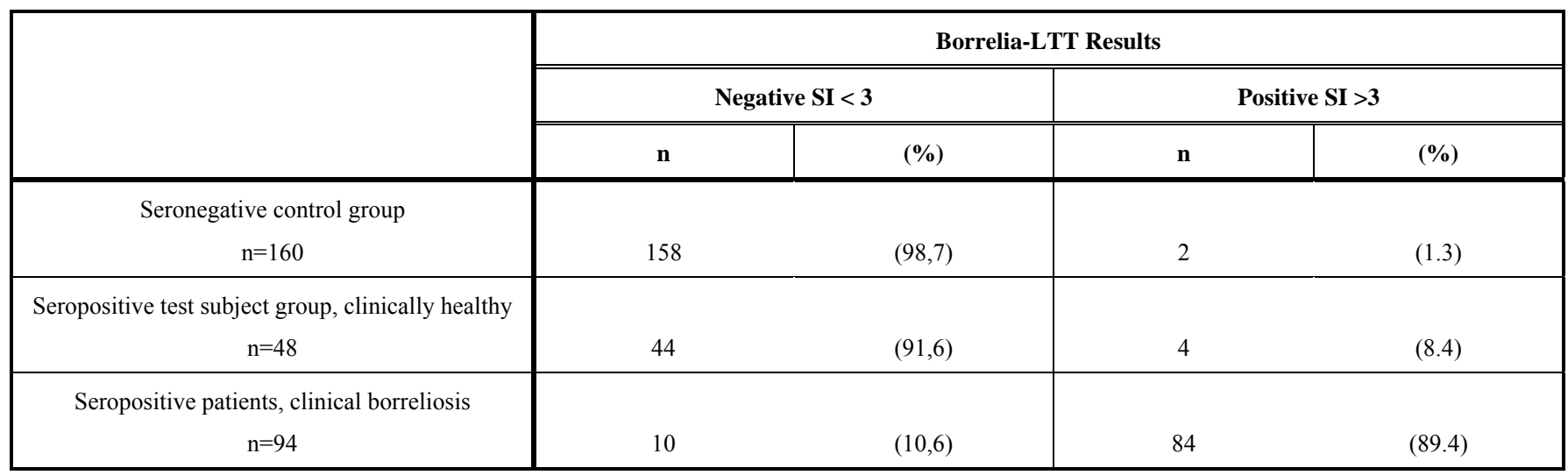


acute neuroborreliosis showed a negative LTT.

In 48 clinically healthy seropositive subjects, $91,6 \%(\mathrm{n}=$ 44) were found to be negative and $8.4 \%(\mathrm{n}=4)$ showed weak positive results in the Borrelia-LTT. The specificity of the positive Borrelia-LTT for clinically active borreliosis was $91.6 \%$ in this group.

Statistical analysis showed a significant difference for the LTT of the Borrelia-IgG/IgM seropositive patients who are clinically active from clinically healthy seropositive patients $(\mathrm{p}<0.0001$, Chi-square test).

\section{Correlation of Borrelia Serology (Immunoblot) and Bor- relia-LTT}

The correspondence of Borrelia serology and BorreliaLTT was studied by re-evaluation of simultaneously conducted laboratory results of 1480 patients with clinical suspicion of borreliosis by parallel determination of the serology and the Borrelia-LTT.

In 1182 out of 1480 patients (79.8\%), the Borrelia serology and Borrelia-LTT showed corresponding results. 37,8\% $(\mathrm{n}=560)$ showed a positive serology and a positive LTT whereas $42 \%(n=622)$ were LTT as well as serology negative. The combination of a positive serology with a negative Borrelia-LTT was found in 266 patients (18.0\%). 32 patients $(2.2 \%)$ were seronegative and LTT-positive.

The discrepant results were followed up retrospectively with the treating physicians in order to clarify any contribution of other clinical conditions (Tables $\mathbf{2 a}$ and $\mathbf{2 b}$ ). The seropositive/LTT-negative group consisted predominantly of patients with status post antibiotic treatment of Lyme borreliosis $(n=240,90,2 \%)$. In the remaining 26 patients only IgM antibodies against OspC were detected. In these cases, there was evidence of rheumatoid arthritis $(\mathrm{n}=15)$, reactive arthritis $(n=3)$ or of a recent EBV infection $(n=5)$.
Among the 32 subjects with the seronegative/LTTpositive constellation, 16 of the patients $(50 \%)$ had early erythema migrans at the time of blood sample collection. The clinical symptoms of the remaining 16 patients were ambiguous - 6 of them had a positive $\mathrm{IgG}$ and another $6 \mathrm{had}$ a positive IgM ELISA test, in each case without the detection of specific bands in the immunoblot. Therefore, $16(1.1 \%)$ of the 1480 Borrelia-LTT results must be regarded as false positives.

The number of false negative results for the Borrelia-LTT can only be determined from the group of 94 seropositive, clinically confirmed borreliosis patients of the validation phase. The rate in these cases was $10.6 \%(\mathrm{n}=10)$.

We would like to mention, that the setting of the study was not suitable to clarify the specificity or sensitivity of the serological tests itself because only the exact clinical evaluation would be suitable as gold standard for this purpose.

\section{Borrelia Lysate Antigens of Three Different Borrelia Species have Proven to be Largely Cross-reactive in the Borrelia-LTT}

340 positive Borrelia-LTT results were evaluated with regard to the number of positive reactions in each individual test using the 3 lysate antigens and rOspC (Table 3). A positive reaction to only one antigen was observed only in rare cases and regarded as unspecific.

The results show that the lysate antigens of all three Borrelia species are predominantly cross-reactive in the Borrelia-LTT.

\section{Positive Borrelia-LTT Declines Significantly Following Antibiotic Treatment}

In addition, follow-up investigations before and 4-6 weeks after a three-week antibiotic treatment were performed in 230 patients with a positive Borrelia-LTT (SI > 5

Table 2a. Analysis of the Constellation of a Positive Borrelia Serology/ Negative LTT

\begin{tabular}{|c|c|c|}
\hline & $\mathbf{n}$ & (\%) \\
\hline \hline Borreliosis after antibiotic therapy (no clinical findings) & 240 & $(90.2)$ \\
\hline Rheumatoid arthritis, LEV & 15 & $(5.7)$ \\
\hline Status post primary EBV infection & 5 & $(1.9)$ \\
\hline Reactive arthritis & 3 & $(1.1)$ \\
\hline unclassifiable & 3 & $(1.1)$ \\
\hline
\end{tabular}

Table 2b. Analysis of the Constellation of a Negative Borrelia Serology/ Positive LTT

\begin{tabular}{|c|c|c|}
\hline & $\mathbf{n}$ & (\%) \\
\hline \hline Erythema migrans & 16 & $(50.0)$ \\
\hline Positive Borrelia ELISA IgM, IgG, immunoblot negative & 12 & $(37.5)$ \\
\hline unclassifiable & 4 & $(12.5)$ \\
\hline
\end{tabular}

$(\mathrm{n}=32)$ 
with 3 Borrelia antigens). 140 of them had a clinical early borreliosis and 90 late manifestations of borreliosis. The division into early $(n=140)$ and late manifestations $(n=90)$ was based on existing clinical data and the probable time of infection, i.e., less than (early borreliosis) or more than one year (late borreliosis) (Table 4).

A significant decrease in SI values in the Borrelia-LTT became apparent after the antibiotic treatment. Among those with early manifestations, $92 \%$ of patients had negative or borderline LTT results 4 to 6 weeks after antibiotic treatment. By contrast, this was true of only $53 \%$ of patients with late manifestations. The retrospective evaluation of clinical data indicated, that in parallel to the LTT, clinical symptoms also regressed significantly or were no longer present. In cases of late manifestations with a persistent positive Borrelia-LTT, partial to complete resistance to treatment of the symptoms was observed.

The serological findings did not change significantly during the same period. In some cases, an increase in the absorbance of the Borrelia ELISA for IgG and IgM antibodies was found. Due to the low sensitivity of the PCR in blood we refrained from performing this parallel analysis in our study.

\section{Follow-up Studies of the Borrelia-LTT Over a Period of One Year}

From the group of early manifestations, 12 patients (ery- thema migrans, $\mathrm{n}=9$; acute neuroborreliosis, $\mathrm{n}=3$ ), and from the group of late borreliosis, 18 patients (Lyme arthritis, $n=12$; chronic neuroborreliosis, $n=6$ ) both the serology and the Borrelia-LTT were performed at least every 3 months in the course of a year. An additional LTT testing is normally performed if a recurrence of Lyme borreliosis is clinically suspected.

The serological findings in all cases showed no significant qualitative changes. Among patients with early manifestations, the Borrelia-LTT that were negative after antibiotic treatment were again weakly positive in only three cases (clinical manifestations were diffuse arthromyalgia and, in one case, painful swelling of an ankle); otherwise, the LTT results in this group were stable negatives (Table 5a ).

Patients with late borreliosis (Table 5b) showed in 16 of 18 cases intermittent multiple increases in SI values, associated with a clinical increase in symptoms in each case. After antibiotic treatment, the SI values decreased again. These "peaks" were also observed after the Borrelia-LTT had become negative.

Only 3 patients (No. 3,11 and 12) showed a reactivation after antibiotic treatment.

\section{DISCUSSION}

In order to investigate the diagnostic value of the Borrelia-LTT, we performed several in vitro investigations and reevaluated patient data and analytical values of patients which

Table 3. Number of Positive Reactions to the 4 Borrelia Antigens in Patients with Positive Borrelia LTT

\begin{tabular}{|c|c|c|}
\hline & $\mathbf{n}$ & (\%) \\
\hline \hline SI $>3$ for all four Borrelia antigens & 162 & $(47.6)$ \\
\hline SI $>3$ in 3 lysate antigens, OspC $<3$ & 76 & $(22.3)$ \\
\hline SI $>3$ in 2 lysate antigens, OspC $>3$ & 63 & $(18.5)$ \\
\hline SI $>3$ in 2 lysate antigens, OspC $<3$ & 24 & $(7.1)$ \\
\hline SI $>3$ in 1 lysate antigen, OspC $>3$ & 15 & $(4.5)$ \\
\hline
\end{tabular}

$(\mathrm{n}=340)$

Table 4. Comparison of Borrelia-LTT in Patients with Early Versus Late Borreliosis Manifestations

\begin{tabular}{|c|c|c|c|}
\hline Early Manifestations $(n=140)$, Before Therapy SI $>5$ & & $\mathbf{n}$ & $(\%)$ \\
\hline \multirow{4}{*}{ after antibiotic therapy } & $\mathrm{SI}<2$ & 109 & $(77.8)$ \\
\hline & $\mathrm{SI}>2<3$ & 20 & $(14.3)$ \\
\hline & SI $>3<5$ & 9 & $(6.4)$ \\
\hline & $\mathrm{SI}>5$ & 2 & (1.5) \\
\hline Late Manifestations ( $\mathrm{n}=90$ ), Before Treatment $\mathrm{SI}>5$ & & $\mathbf{n}$ & $(\%)$ \\
\hline \multirow{4}{*}{ after antibiotic therapy } & $\mathrm{SI}<2$ & 20 & $(223)$ \\
\hline & $\mathrm{SI}>2<3$ & 28 & $(31.0)$ \\
\hline & $\mathrm{SI}>3<5$ & 32 & $(35.6)$ \\
\hline & $\mathrm{SI}>5$ & 10 & $(11.1)$ \\
\hline
\end{tabular}

(SI $<2$ =negative; $\mathrm{SI}>2<3=$ borderline, $\mathrm{SI}>3<5$ = weak positive; $\mathrm{SI}>5$ = positive) 
Table 5a. Borrelia-LTT Follow-Up Studies (Early Manifestations)

\begin{tabular}{|c|c|c|c|c|}
\hline Month & $\mathbf{0}$ & $\mathbf{3}$ & $\mathbf{6}$ & $\mathbf{9}$ \\
\hline \hline patient 1 & 6.8 & $<2^{*}$ & $<2$ & $<2$ \\
\hline patient 2 & 9,6 & $<2^{*}$ & $<2$ & $<2$ \\
\hline patient 3 & 10.9 & $<2^{*}$ & $<2$ & $<.8$ \\
\hline patient 4 & 12.6 & $<2^{*}$ & $<2$ & $<2$ \\
\hline patient 5 & 10.2 & $<2^{*}$ & $<2$ & $<2$ \\
\hline patient 6 & 8.4 & $<2^{*}$ & $<2$ & $<2$ \\
\hline patient 7 & 11.8 & $<3^{*}$ & $<2$ & $<2$ \\
\hline patient 8 & 8.8 & $<2^{*}$ & $<2$ & $<2$ \\
\hline patient 9 & 19.6 & $<3^{*}$ & $<3$ & $<2$ \\
\hline patient 10 & 13.4 & $<3^{*}$ & $<2$ & $<2$ \\
\hline patient 11 & 6.5 & $<2^{*}$ & $<2$ & $<2$ \\
\hline patient 12 & 10.2 & $<3^{*}$ & $<.8$ & $<2$ \\
\hline
\end{tabular}

(* 4-6 weeks after antibiotic therapy)

Shown are the results of follow-up studies with the Borrelia-LTT for 12 patients with early manifestations of borreliosis (mainly E. migrans) over a period of 1 year from the date of initial diagnosis before therapy (the highest of the 4 respective SI values of the Borrelia-LTT is given).

Only 3 patients (No. 3,11 and 12) showed a reactivation after antibiotic treatment.

Table 5b. Borrelia-LTT Follow-up Studies (Late Manifestations)

\begin{tabular}{|c|c|c|c|c|c|}
\hline Month & 0 & 3 & 6 & 9 & 12 \\
\hline patient 2 & 9.4 & $3.3^{*}$ & $<2 *$ & 5.8 & $<2 *$ \\
\hline patient 3 & 9.8 & $4.8^{*}$ & $<2 *$ & $6.2^{*}$ & $3.9 *$ \\
\hline patient 4 & 10.6 & $5.2 *$ & $3.5^{*}$ & $<2 *$ & 6.4 \\
\hline patient 6 & 16.2 & $6.0^{*}$ & $3.2^{*}$ & 7.2 & $4.8^{*}$ \\
\hline patient 7 & 14.8 & $<3 *$ & $<2$ & 4.9 & $<2 *$ \\
\hline patient 8 & 10.8 & $6.2 *$ & $3.8^{*}$ & 8.2 & $3.6^{*}$ \\
\hline patient 9 & 6.3 & $<2 *$ & $<2$ & $<2$ & $<2$ \\
\hline patient 13 & 7.8 & 4.8 & $4.2^{*}$ & $<3^{*}$ & 3.8 \\
\hline patient 14 & 6.9 & $<2 *$ & 3.6 & $<2 *$ & 4.9 \\
\hline patient 15 & 9.6 & $<2 *$ & 5.4 & $<2 *$ & 3.1 \\
\hline patient 16 & 12.1 & 4.2 & $3.1^{*}$ & $<2 *$ & $<2$ \\
\hline patient 17 & 14.4 & 5.4 & $4.8^{*}$ & $<3 *$ & 4.5 \\
\hline patient 18 & 10.6 & $<3^{*}$ & $<2$ & $<2$ & $<2$ \\
\hline
\end{tabular}

(* 4-6 weeks after antibiotic therapy)

Shown are the results of follow-up studies with the Borrelia-LTT over a period of 1 year in 18 patients with late manifestations of borreliosis (an infection more than 2 years previously). Only 3 patients (No. 5, 9 and 10) showed no reactivations after initial treatment with antibiotics.

were investigated routinely in our laboratory.

A Borrelia-LTT with one recombinant antigen and lysate antigens of the three relevant Borrelia species (B. sensu stric- to, B. afzelii and B.garinii) was developed and tested. The results achieved thus allow us to answer the following questions: 
1. In patients with clinical borreliosis prior to the start of antibiotic therapy, there is a high degree of correspondence between the results of Borrelia serology and Borrelia-LTT studies.

2. The sensitivity of the Borrelia-LTT is $89.4 \%$ for clinically active borreliosis, with a specificity of $98,7 \%$.

3. The lysate antigens of the three species of Borrelia and the recombinant OspC cross-reacted in the Borrelia-LTT. Therefore, it is not possible to determine the respective species involved.

4. The negative results in clinically healthy seropositive subjects and the studies before and after antibiotic treatment of patients with clinically active disease are a strong indication that the Borrelia-LTT with lymphocytes from peripheral blood is positive only when the immune system is currently being stimulated by Borrelia. The proof that the test responds only during an active Borrelia infection could only be provided by the simultaneous detection of Borrelia by culture or Borrelia PCR (Borrelia DNA detection). In our patient cohort, this was demonstrated in only 6 out of the 32 cases tested by Borrelia PCR.

The results of our study differ in part from some published data which show a low specificity of the Borrelia-LTT $[24,25]$. This is very likely due to methodology. The addition of interferon- $\alpha$ to the cell culture medium inhibits nonspecific proliferation of lymphocytes and promotes the function of antigen-presenting cells. This improves the discriminatory power of positive and negative LTT results, even though the SI values of the positive reactions and the blank values are lower than in assays without interferon [23]. Another modification is the use of polymyxin B for the elimination of nonspecific activating lipid groups from the Borrelia lysates and traces of LPS from the rOspC expressed in E. coli. In this way, common, nonspecific borderline and weak positive LTT reactions were eliminated (data not shown).

Of great importance are the selection and especially the dosage of the Borrelia test antigens. Lysate antigens, kindly provided by Seramun (Heidesee), were specially purified for the ELISA test and showed no positive reactions with negative control sera. Nevertheless, the presence of Borrelianonspecific proteins in the lysates that may cross-react with other bacterial species may be unavoidable.

Our own experience in the development of antigenspecific LTT applications show that the "specific diagnostic width" of the test antigens is particularly important. For the Borrelia-LTT, therefore, it was necessary to consider whether those concentrations of Borrelia test antigens which cause barely any positive/borderline LTT reactions in 20 seronegative subjects, are sufficient to detect Borrelia-specific helper cells in the blood of patients with clinical borreliosis.

Obviously, the advantage of the Borrelia-LTT presented here is the use of a mixture of Borrelia-specific antigens in the Borrelia lysates. This is confirmed by the calculated sensitivity of $89,6 \%$ and specificity of about $98,7 \%$ for seropositive clinical borreliosis prior to antibiotic therapy. In contrast, in preliminary tests with all recombinant Borrelia proteins (p93, p39, p34, p25, p18) available to us, only the rOspC (p25) was proven to be a suitable test antigen for the Borrelia-LTT. For all other proteins, the "specific diagnostic width" was too small (results of these preliminary tests are not shown). Nevertheless, the use of lysate antigens in LTT studies is, in principle, problematic, since each new antigen batch has to be tested in parallel with the previously used antigen batch. Therefore, the studies presented here were carried out with two different batches of antigen, which was especially important for the LTT results. The results achieved so far with lysate antigens, however, encourage us to test mixtures of recombinant antigens or Borrelia antigens purified from lysates in the LTT in the future in order to achieve well-defined reproducible test antigens.

A strong argument for the Borrelia-specific LTT reactions to the test antigens used is the fact that the positive reactions following antibiotic treatment were always regressive, and in early manifestations of Lyme borreliosis, they were predominantly negative. These results correspond to observations of other authors $[12,14,16,21]$, but also contradict other reports $[24,25,26]$. The discrepancies are very likely due to the LTT method modified by us and the different antigens. According to our results, it is very likely that Borrelia-specific $\mathrm{T}$ helper cells, after overcoming the active infection (i.e., elimination of Borrelia antigens), migrate mainly from the bloodstream into the lymph nodes or spleen, and therefore their numbers in the blood are lower than the detection limit for the LTT. An allergen elimination test has the same effect in appropriate LTT applications in patients with type IV allergies to medications (non-published personal observation). Since the total number of $\mathrm{T}$ cells in the blood is relatively constant in individuals and even decreases with age, it is justified to assume that Borrelia-specific $\mathrm{T}$ cells circulate only during an active confrontation of the immune system with appropriate antigens in large numbers in the blood.

A Borrelia-LTT test that has become negative is not evidence, however, that a Borrelia infection has been cured. This was demonstrated in follow-up studies over a one year period in patients with late borreliosis. Borrelia that persist in spite of antibiotic treatment, which have been described multiple times $[27,28,29,30]$, are very likely responsible for this phenomenon. After reactivation of the infection with renewed antigen presence, apparently Borrelia-specific $T$ cells are rapidly mobilized from the lymphoid organs. The clinical symptoms and the course of the LTT reactions suggest that these patients could suffer from persistent or latent borreliosis with phases of reactivation. For this reason doubts are arising about the prevailing opinion that a single, concerning dose and duration appropriate, antibiotic treatment is sufficient to eliminate the Borrelia completely.

We were able to confirm an important piece of information from Krause et al [12] on the beneficial effect of autologous serum for Borrelia-LTT. It is very likely that the antibodies contained in the serum form immune complexes with Borrelia antigens and thus promote uptake by antigenpresenting cells.

In summary, the following conclusions can be made for the use of the Borrelia-LTT:

1. Except for early manifestations of borreliosis (2 to 6 weeks after infection), the detection of Borrelia-specific antibodies to confirm the clinical diagnosis of borreliosis is 
often sufficient. Only in the case of an unclear clinical picture of borreliosis coupled with negative or borderline serology the Borrelia-LTT can be beneficial in order to make a decision regarding the indication for antibiotic treatment since it also exhibits a clear positive reaction in the case of early manifestations.

2. In the case of infections in the more remote past with an ambiguous clinical picture and positive Borrelia serology, the Borrelia-LTT provides an important indication as whether an active Borrelia infection could exist. The Borrelia serology is not suitable in this case, and the direct pathogen detection methods (PCR or culture) are also not sufficient for answering this question due to their low sensitivity. It must be noted, however, that it is not only the results of the Borrelia-LTT that are important for the indication for antibiotic treatment, but also the medical history and the current clinical picture.

3. It has been shown that the Borrelia-LTT can be used to evaluate the success of antibiotic treatment, although the clinical course is also particularly important. However, the symptoms of disseminated borreliosis may persist after successful treatment for some time, however. An LTT follow-up examination is reasonable, at the earliest, 4 to 6 weeks after the completion of therapy. This interval is necessary because, on the one hand, possibly surviving Borrelia can become active during this time and, on the other hand, Borrelia-specific $\mathrm{T}$ cells persist in the blood for some time after elimination of the antigens. The Borrelia-LTT should not be performed during antibiotic therapy because, in our experience, the result will be negative, but soon after discontinuation of treatment, it may again become positive.

Criticism is often based on the frequency of falsepositive results of the Borrelia-LTT (5). This problem continues to exist unless there is sufficient testing of the antigen specificity of the Borrelia-LTT. In the case of frequently false positive reactions, the dose of the test antigens is too high. Currently, since there is no available gold standard for the laboratory diagnosis of borreliosis or its exclusion, the Borrelia-LTT can only be validated according to clinical and serological findings. Nevertheless, there are still gaps in the validation that we conducted. For instance, it was only possible in individual cases to examine patients with active syphilis $(\mathrm{n}=3)$ or leptospirosis infection $(\mathrm{n}=2)$ for potential cross reactivity. In these few cases, there was no evidence of such cross-reactivity in the Borrelia-LTT. Allergies, autoimmune diseases and acute, persistent and latent viral infections (including HIV, EBV, CMV, VZV) have now been excluded, by further investigations, as a possible cause of false-positive reactions (unpublished data).

The Borrelia-LTT cannot provide any information on whether a patient has ever had a Borrelia infection. This question is largely answered only by serology. Our studies presented here and the results of other authors $[12,14,16$, 21], particularly the work of Valentine-Thon et al. on LTTMELISA [31], provide good arguments that the positive Borrelia-LTT indicates an active borreliosis which, however, could only be definitely proven in conjunction with positive detection of Borrelia. However, we succeeded in doing so in only 3 seronegative patients with erythema migrans using positive Borrelia-PCR in the blood (unpublished data).
The in vitro antigen-induced lymphocyte proliferation is the approved cellular immunological method for detecting antigen-specific memory $\mathrm{T}$ helper cells. In addition and prior to clonal lymphocyte proliferation, however, a series of immunologically relevant genes for both cell surface markers and especially cytokines such as interleukin-2 and interferon$\gamma$ are activated, which in turn are used as the basis for newer cellular immunological laboratory methods. An example is the QuantiFERON test (interferon- $\gamma$ stimulation) performed to detect a Mycobacter tuberculosis infection [32]. However, a distinction between a latent and florid infection is not possible with this test, however. Currently, the ELISPOT test (quantitative determination of cytokine-producing lymphocytes) is used in vaccine research in particular, and is also being tested in patients with a Borrelia infection. According to the previously published results by Forsberg et al. and our own experiences, even though seropositive individuals are detected by the ELISPOT assay, a differentiation between symptomatic and asymptomatic Borrelia infections was not possible [33-35]. The advantage of antigen-specific cytokine-stimulation assays would be that, while 6 days are required for an antigen-specific LTT test, the incubation period for the cytokine assay is only about 24 hours. It should be noted, however, that the biology on which these cellular immunological test systems are based is very different. While in the LTT setting T helper memory cells are clearly identified as cytokine producers, also other lymphocyte subpopulations like NK cells, and (depending on the cytokine) also monocytes are being taken into consideration as cytokine producers. However, cellular short-term stimulation methods are rather sensitive to nonspecific activations, while, in the LTT such influences dominate only during the first 48 to 72 hours, and thus have virtually disappeared after 6 days at the time the cellular proliferation is measured. For the particular issue of florid or non-active Borrelia infection, the situation is even more difficult since there is usually no gold standard available (i.e., patients with positive detection of live Borrelia). In this difficult situation, a new method should always be validated in comparison with an extensively proven method. The aim of our investigations were therefore to evaluate the results previously obtained with an optimized Borrelia-LTT in order to provide a basis for necessary comparative prospective studies with cellular immunological methods based on antigen-specific gene activation or cytokine stimulation. In our opinion, the Borrelia-LTT presented here fulfills the associated requirements.

Finally, it should be emphasized again that the primacy for the diagnosis of borreliosis remains with the medical history and clinical symptoms. In second place is the determination of Borrelia-specific antibodies in the blood or cerebral synovial fluid. With the Borrelia-LTT another diagnostic tool is available that can help to clarify issues for the indication of antibiotic treatment. The Borrelia-LTT is particularly important, however, for assessing the effects of therapy if the initial findings are positive, as they should prove negative four weeks after the end of therapy, or at least should prove to have declined significantly.

\section{CONFLICT OF INTEREST}

The authors confirm that this article content has no conflicts of interest. 


\section{ACKNOWLEDGEMENTS}

None declared.

\section{REFERENCES}

[1] Steere AC. Lyme disease. N Engl J Med 2001;345:115-25.

[2] Tewald F, Braun R. Durchführung und Interpretation serologischer Tests bei Verdacht auf Borrelieninfektion. Clin Lab 1998; 44: 897902.

[3] Dejmkova H, Hulinska D, Tegzova D, Pavelka K, Gatterova J, Vavrik P. Seronegative Lyme arthritis caused bei Borrelia garinii. Clin Rheumatol 2002; 21: 330-4.

[4] Aguero-Rosenfeld ME, Wang G, Schwartz I, Wormser GP. Diagnosis of Lyme borreliosis. Clin Microbiol Rev 2005; 18: 484-509.

[5] Wilske B, Fingerle V. Lyme-Borreliose Diagnostik. Mikrobiologe 2005; 15: 209-20.

[6] Kalish RA, McHugh G,Granquist J, Ruthazer R, Steere AC. Persistence of immunoglobulin $\mathrm{M}$ or $\mathrm{G}$ antibody responses to Borrelia burgdorferi 10-20 years after active Lyme disease. Clin Infect Dis 2001; 33: 780-5.

[7] Schütt C. Lymphozytentransformationstest (LTT). In:Friemel H, Ed. Immunologische Arbeitsmethoden. Stuttgart: G. Fischer Verlag Jena 1991; pp. 349-55.

[8] Sigal LH, Moffat CM, Steere AC, Dwyer JM. Cellular immune findings in Lyme disease. Yale J Biol Med 1984; 57: 595-8.

[9] Sigal LH, Steere AC, Freeman DH, Dwyer JM. Proliferative responses of mononuclear cells in Lyme disease. Arthritis Rheum 1986; 29: 761-9.

[10] Dattwyler RJ, Volkman DJ, Luft BJ, Halperin JJ, Thomas J, Golightly MG. Seronegative Lyme disease. N Engl J Med 1988; 319: 1441-6.

[11] Dressler F, Yoshinari NH, Steere AC. The T-cell proliferative assay in the diagnosis of Lyme disease. Ann Intern Med 1991; 115: 533-9.

[12] Krause A, Brade V, Schoerner C, Solbach W, Kalden JR, Burmester GRT cellproliferation induced by Borrelia burgdorferi in patients with Lyme borreliosis. Arthritis Rheum 1991; 34: 393-402.

[13] Schempp C, Owsianoski M, Lange R, Gollick H. Comparison of Borrelia burgdorferi ultrasonicate and whole B. burgdorferi cells as a stimulus for T-cell proliferation and GM-CSF secretion in vitro. Zentralbl Bakteriol 1993; 279: 417-25.

[14] Schempp C, Bocklage H, Owsianowski M, Lange R, Orfanos CE, Gollnick H. In vivo und in vitro Nachweis einer Borrelieninfektion bei einer morpheaähnlichen Hautveränderung mit negativer Borrelienserologie Hautarzt 1993; 44: 14-8.

[15] Buechner SA, Lautenschläger S, Itin P, Bircher A, Erb P. Lymphoproliferative responses to Borrelia burgdorferi in patients with erythema migrans, acrodermatitis chronica atrophicans, lymphadenosis benigna cutis, and morphea. Arch Dermatol 1995; 131: 673-7.

[16] Breier F, Klade H, Stanek G, Poitschek C, Kirnbauer R, Dorda W, Aberer E. Lymphoproliferative responses to Borrelia burgdorferi in circumscribed scleroderma. Br J Dermatol 1996; 134: 285-91.

[17] Rutkowski S, Busch DH, Huppertz HI. Lymphocyte proliferation assay in response to Borrelia burgdorferi in patients with Lyme arthritis:analysis of lymphocyte subsets. Rheumatol Int 1997; 17: 151-8.

[18] Zoschke DC, Skemp AA, Defosse DL. Lymphoproliferative responses to Borrelia burgdorferi in Lyme disease. Ann Intern Med 1991; 114: 285-9.

[19] Roessner K, Fikrig E, Russel JQ, Cooper SM, Flavell RA, Budd RC. Prominent T lymphocyte response to Borrelia burgdorferi from peripheral blood of unexposed donors. Eur J Immunol 1994; 24: 320-4.

Huppertz HI, Mösbauer S, Busch DH, Karch H. Lymphoproliferative responses to Borrelia burgdorferi in the diagnosis of Lyme arthritis in children and adolescents. Eur J Pediatr 1996; 155: 297 302.

[21] Breier F, Klade H, Stanek G. Lymphoproliferationstest bei kutanen Manifestationen der Lyme-Borreliose. Wien Med Wochenschr 1995; 27: 170-3.

[22] Boyum A. Isolation of mononuclear cells and granulocytes from human blood. Scand J Cin Lab Invest 1968; (Suppl 97):77.

[23] von Baehr V, Mayer W, Liebenthal C, von Baehr R, Bieger W, Volk HD. Improving the in vitro antigen specific $\mathrm{T}$ cell proliferation assay: the use of interferon-alpha to elicit antigen specific stimulation and decrease bystander proliferation. J Immunol Methods 2001; 251: 63-71.

[24] Horowitz HH, Pavia CS, Bittker S, et al. Sustained cellular immune response to Borrelia burgdorferi: Lack of correlation with clinical presentation and serology. Clin Diagn Lab Immunol 1994; 1: 373 8.

[25] Pohl-Koppe A, Kaunicnik A, Wilske B. Characterization of the cellular and humoral immune response to outer protein $\mathrm{C}$ and outer protein 17 in children with early disseminated Lyme borreliosis. Med Microbiol Immunol 2001; 189: 193-200.

[26] Vaz A, Glickstein L, Field JA, et al. Cellular and humoral immune responses to Borrelia burgdorferi antigens in patients with culturepositive early Lyme disease. Infect Immun 2001; 69: 7437-44.

[27] Priem S, Burmester GR, Kamstadt T, Wolbart K, Rittig MG, Krause A. Detection of Borrelia burgdorferi by PCR in synovial membrane, but not synovial fluid from patients with persisting Lyme arthritis after antibiotic therapy. Ann Rheum Dis 1998; 57: 118-21.

[28] Phillips SE, Mattman LH, Hulinska D, Moayad H. A proposal for the reliable culture of Borrelia burgdorferi from patients with chronic Lyme disease, even from those previously aggressively treated. Infektion 1998; 26: 364-7.

[29] Oksi J, Marjamaki M, Nikoskelainen J, Viljanen MK. Borrelia burgdorferi detected by culture and PCR in clinical relapse of disseminated Lyme borreliosis. Ann Med 1999; 31: 225-32.

[30] Honegr K, Hulinska D, Beran J, Dostal V, Havlasova J, Cermakova Z. Long term and repeated electron microskopy and PCR detection of Borrelia burgdorferi sensu lato after an antibiotic treatment. Cent Eur J Public Health 2004; 12: 6-11.

[31] Valentine-Thon E, Ilsemann K, Sandkamp M. A novel lymphocyte transformation test (LTT-MELISA) for Lyme borreliosis. Diagn Microbiol Infect Dis 2007; 57: 27-34.

[32] Connell TG, Rangaka MX, Curtis N, Wilkinson RJ. QuantiFERON-TB Gold: state of the art for the diagnosis of tuberculosis infection? Expert Rev Mol Diagn 2006; 6: 663-77.

[33] Forsberg P, Ernerudh J, Ekerfelt C, Roberg M, Vrethem M, Bergström S. The outer surface proteins, of Lyme disease borrelia spirochetes stimulate $\mathrm{T}$ cells to secrete interferon-gamma (IFNgamma): diagnostic and pathogenic implications. Clin Exp Immunol 1995; 101: 453-60.

[34] Ekerfelt C, Forsberg P, Svenvic M, Roberg M, Bergström S, Ernerudh J. Asymptomatic borrelia-seropositive individuals display the same incidence of Borrelia-specific interferon-gamma (IFNgamma)-secreting cells as patients with clinical Borrelia infection. Clin Exp Immunol 1999; 115: 498-502.

[35] Ekerfelt C, Masreliez C, Svenvik M, Ernerudh J, Roberg M, Forsberg $\mathrm{P}$. Antibodies and T-cell reactivity to Borrelia burgdorferi in an asymptomatic population: a study of healthy blood donors in an inland town district in the south-east of Sweden. Scand J Infect Dis 2001; 33: 806-8.

This is an open access article licensed under the terms of the Creative Commons Attribution Non-Commercial License (http:/creativecommons. org/licenses/by-nc/3.0/) which permits unrestricted, non-commercial use, distribution and reproduction in any medium, provided the work is properly cited. 